\title{
Vom historischen Erbe zur selbstbestimmten Sprach(en)- politik? Literarische Mehrsprachigkeit in Litauen und Lettland
}

\author{
NATALIA BLUM-BARTH
}

\begin{abstract}
From Historical Legacy to Self-Determined Language(s) Policy? Literary Multilingualism in Lithuania and Latvia. The first part of this article looks at Soviet language(s) policy. Two further parts discuss language(s) policy and literary multilingualism in Lithuania and Latvia. The aim is not to provide a differentiated investigation, but to show similarities and differences as well as tendencies in the language(s) politics of the two states from the $19^{\text {th }}$ century to the present in the mirror of literature and to explain them using case studies. In the fourth, concluding part, literary translation is highlighted as one of the formats for implementing multilingualism outside the text with particular focus on the consultative function of the Russian language. ${ }^{1}$
\end{abstract}

Keywords: literary multilingualism; language(s) policy; translation; Lithuania; Latvia; Soviet Union

1 Sowjetische Sprach(en)politik und ihre Auswirkungen auf die

\section{Literatur}

In der „Deklaration der Rechte der Völker Rußlands“2, einem der ersten Dokumente, das die Bolschewiki nach ihrer Machtübernahme bereits am 2. (15.) November 1917 verabschiedeten, wurde der Grundsatz der nationalen Politik festgelegt: Die Gleichberechtigung aller Nationen, einschließlich das Recht auf den Gebrauch der Muttersprache der jeweiligen nationalen

1 In Anlehnung an Patrick Schreiner, der Sprachenpolitik als „das Verhältnis zwischen mindestens zwei Sprachen in funktionaler, politischer und territorialer Hinsicht" definiert (Schreiner 2006: 26), wird in diesem Beitrag die Schreibweise Sprach(en) politik verwendet, um sowohl auf die Hierarchisierung der Sprachen im literarischen Schreibprozess hinzuweisen, als auch die politisch gesteuerte Bevorzugung einer der Sprachen in einer mehrsprachigen Gesellschaft zum Ausdruck zu bringen.

2 Аекцарация прав народов России: www.hist.msu.ru/ER/Etext/DEKRET/peoples. htm. 
BLUM-BARTH

Minderheit. Auch wenn dieser Grundsatz in der Phase der Industrialisierung 1928-1931 zugunsten der russischen Sprache als lingua franca aufgegeben wurde, wurden in der ersten Hälfte der 20er Jahre einzigartige Maßnahmen ergriffen, um Sprachen der nationalen Minderheiten und Ethnien voneinander abzugrenzen, zu entfalten und zu reformieren. Die Aufmerksamkeit galt in erster Linie den Sprachen und Dialekten von Kirgisen, Baschkiren, Turkmenen, Usbeken, Tadschiken, Tataren, Aserbaidschanern, Dagestanern und Kareliern. Einige dieser Sprachen bekamen zum ersten Mal ein Alphabet, viele dieser Sprachen wurden auf das lateinische Alphabet umgestellt, Linguisten erstellten Grammatik- und Lehrbücher für den Schulunterricht (Borisova o.J.: 4f). Diese Aufwertung der Dialekte und Regionalsprachen fand im Rahmen der ,Verwurzelungspolitik' (vgl. Chalitova 2010: 156) der Bolschewiken statt. Um das Ziel, die ideologische Indoktrinierung, zu erreichen, bediente man sich lokaler Sprachen. Ihre Herausbildung zum Rang der Literatursprachen sollte die Akzeptanz der bolschewistischen Ideen, die Ausbildung der loyalen Anhänger aus den Reihen der autochthonen Bevölkerung und den Ausbau der sozialistischen Gesellschaft befördern.

1934 markiert den Wendepunkt in der Sprachpolitik der Sowjetunion. Dieser zeichnet sich durch die zunehmende Verbreitung der russischen Sprache in den nationalen Minderheiten der Sowjetunion aus (Borisova o.J.: 10). Am 13. März 1938 wird Russisch zum Pflichtfach in allen Schulen der Sowjetunion. Das zentral koordinierte Programm soll die Implementierung des Russischunterrichts in allen Schulen und Bildungseinrichtungen aller Republiken der Sowjetunion steuern. Der Erfolg dieses Programms wurde durch seine großzügige Finanzierung durch das Zentralkomitee garantiert. Außerdem wird Russisch die einzige Sprache der Roten Armee. Dadurch wird der Wehrdienst zum Instrument der Russifizierung, was auch eine bessere Kenntnis der russischen Sprache in der männlichen Bevölkerung der nationalen Minderheiten erklärt. Bereits Anfang 1940 kann die faktische Dominanz der russischen Sprache als offizielle Sprache in den meisten Republiken der Sowjetunion konstatiert werden. Eine Ausnahme stellen die baltischen Staaten dar. Anders als in allen anderen sowjetischen Republiken haben sich die jeweiligen Landessprachen erfolgreich gegen die russische Sprache durchgesetzt. Veranschaulichen lässt sich dies anhand der Statistik über die Buchveröffentlichungen aus dem Jahr 1940 (Borisova o.J.: 24). 
Vom historischen Erbe zur selbstbestimmten Sprach(en)politik?

\begin{tabular}{l|c|c}
\hline Republik & $\begin{array}{c}\text { Neuerscheinungen } \\
\text { insgesamt }\end{array}$ & $\begin{array}{c}\text { Neuerscheinungen in der } \\
\text { Landessprache }\end{array}$ \\
\hline Litauen & 387 & 336 \\
Lettland & 392 & 286 \\
Estland & 266 & 229 \\
Ukraine & 4836 & 2012 \\
Weißrussland & 772 & 375 \\
Kasachstan & 762 & 382 \\
\hline
\end{tabular}

Wenn 1940 in Litauen und Estland 87 \% der Bücher und in Lettland knapp $73 \%$ der Neuerscheinungen in der jeweiligen Landessprache veröffentlicht wurden, so waren es in der sowjetischen Ukraine knapp $42 \%$, in Weißrussland $48 \%$ und in Kasachstan $50 \%$. Damit scheint die Sprachsituation in den baltischen Staaten der Sowjetzeit eine deutlich andere zu sein als in anderen sowjetischen Republiken. Während das Russische in der sowjetischen Ukraine und im sowjetischen Weißrussland die Landessprachen aus dem Buchdruck immer mehr verdrängte, konnten Lettisch, Estnisch und Litauisch weiterhin den Status der Bildungssprache aufrechterhalten.

In den 60er Jahren wurde die russische Sprache zur zweiten Muttersprache jedes nichtrussischen Bürgers der Sowjetunion erklärt. Die tatsächlichen Kenntnisse der russischen Sprache spielten dabei keine Rolle. ${ }^{3}$ Um den Russischunterricht zu fördern, wurde Russisch 1975 als Pflichtfach ab der ersten Klasse in den Schulen von neun sowjetischen Republiken eingeführt, in den übrigen Republiken war es Pflichtfach ab der zweiten Klasse (Rudnev 2007: 4). Die letzten sowjetischen Republiken, die den Russischunterricht ab dem ersten Schuljahr einführten, waren Litauen und Estland. Dies geschah erst $1980 !^{4}$

Dass die baltischen Republiken ihre Landessprachen stärken und dem Einfluss des Russischen Widerstand leisten konnten, hängt nicht zuletzt mit der moderaten ideologischen Indoktrinierung im Baltikum im Vergleich $\mathrm{zu}$ den anderen sowjetischen Republiken zusammen. Im Bereich der Literatur kann man dies an Laureaten des Gorki Staatspreises veranschaulichen (Государственная премия РСФСР имени М. Горького). Dieser Literaturpreis wurde von 1966 bis 1991 vom Ministerrat jährlich an drei bzw. vier

3 Laut Volkszählung 1989 gaben beispielsweise 36,6\% der Letten, knapp 40\% der Kasachen und ca. 53\% Armenier an, dass sie keine Russischkenntnisse besitzen. Vgl. Rudnev 2007: 5.

4 Ebd. 
Literaten verliehen und galt als eine der höchsten literarischen Auszeichnungen der Sowjetunion. Mit dem Gorki Staatspreis wurden ideologisch geprägte Werke ausgezeichnet, die den Bau des Sozialismus und den Fortschritt der Planwirtschaft in höchsten Tönen lobten. Fast jedes Jahr war unter den Ausgezeichneten auch ein nichtrussischer sowjetischer Autor. Dadurch wollte man der multinationalen und mehrsprachigen Situation in der Sowjetunion gerecht werden. Unter den Preisträgern aller Jahrgänge finden sich keine Autoren aus den baltischen Ländern.

Unter den Autoren der baltischen Republiken der Sowjetzeit war die Aufgabe der Muttersprache als Sprache der Kreativität zugunsten des Russischen äußerst selten. ${ }^{5}$ Selbst die Autorinnen und Autoren, die in ihren Werken der kommunistischen Ideologie Tribut leisteten, schrieben weiterhin in ihren Muttersprachen. Für die baltischen Autorinnen und Autoren scheint die textübergreifende/textexterne Mehrsprachigkeit kennzeichnend zu sein.

Georg Kremnitz unterscheidet zwischen der Ebene des Textes (textintern) und der Ebene des Gesamtwerkes des Autors (textübergreifend): „Während in der ersten Form innerhalb eines Textes mehrere Sprachen verwendet werden, benützt in der zweiten Form ein Autor in unterschiedlichen Texten verschiedene Sprachen." (Kremnitz 2015: 18) Bei der textübergreifenden Mehrsprachigkeit ist also die Mehrsprachigkeit der Autorinnen und Autoren gemeint, bei der textinternen - die Mehrsprachigkeit des Textes. Die Mehrsprachigkeit des Autors führt nicht automatisch zur Mehrsprachigkeit des Textes (Blum-Barth 2019: 12). Zu den zwei von Kremnitz definierten Realisierungsformaten der textübergreifenden Mehrsprachigkeit - 1. das schriftstellerische Euvre, das in zwei oder mehr Sprachen vorliegt, ${ }^{6}$ und 2. Selbstübersetzungen - können auch 3. literarische Fremdübersetzungen gezählt werden. ${ }^{7}$

In der textexternen literarischen Mehrsprachigkeit baltischer Autorinnen und Autoren dominieren Fremdübersetzungen, und zwar von Werken aus dem Kanon der Weltliteratur. Unabhängig vom Realisierungsformat schlägt

5 Der Sprachwechsel und die Zweisprachigkeit sind in erster Linie für die Autoren des Nordostens und Nordens der Sowjetunion kennzeichnend. Zeitlich fällt diese Entwicklung in die 70er und 80er Jahre und korrespondiert somit mit den Assimilierungsprozessen und der Aufgabe der Muttersprachen dieser Völker zugunsten der russischen Sprache.

6 Schriftsteller wie Nabokov, Beckett, Goll, Goldschmidt, Schickele, Ionesco, Filip, Semprún u.a.

7 Diese sind besonders im Bereich der Lyrik verbreitet. Paul Celan, der sich für seine Dichtung keine andere Sprache außer seiner deutschen Muttersprache vorstellen konnte, übersetzte aus neun Sprachen ins Deutsche. Vgl. Gellhaus 1997. 
Vom historischen Erbe zur selbstbestimmten Sprach(en)politik?

sich in der textübergreifenden/textexternen Mehrsprachigkeit die Reaktion der Autorinnen und Autoren auf die Sprach(en)politik nieder, so dass dadurch ihr Versuch dokumentiert wird, eigene literarische Tätigkeit mit den sprachpolitischen und sprachsoziologischen Rahmenbedingungen zu vereinbaren.

\section{Sprach(en)politik und literarische Mehrsprachigkeit in Litauen}

Kennt man die Geschichte Litauens, so erschließt sich von selbst, dass in diesem von Nachbarländern geteilten und beherrschten Land mehrere Ethnien und Sprachen zu Hause waren. Mit der Dritten Polnischen Teilung 1795 bildeten der Südosten Litauens und das nordwestliche Grenzgebiet Weißrusslands das Gouvernement Wilna, in dem Russisch, Litauisch, Polnisch, Weißrussisch sowie Hebräisch und Jiddisch gesprochen wurden. Außerdem gab es ethnische Minderheiten wie Deutsche und Tataren, die im Alltag ihre Muttersprachen verwendeten. Wie in den meisten multiethnischen und vielsprachigen Gebieten $\mathrm{zu}$ beobachten ist, gab es auch in Litauen eine Hierarchie der Sprachen (vgl. Kostiucenko 2016), die auf die Literatur des Landes abfärbte. Die Sprache der literarischen Texte wurde nicht zuletzt durch die Sprache des Schulunterrichts vorgegeben. Denn die Prägung durch literarische Texte im Schulunterricht und die literarische Vorbildung sind meistens bestimmend für die ersten literarischen Versuche junger Autorinnen und Autoren. Erst im Verlauf ihrer literarischen Tätigkeit stellt sich die Frage nach der Wahl bzw. nach der Entscheidung für die eine oder andere Sprache für ihre kreative Tätigkeit. Die litauische Literatur des 19. Jahrhunderts liefert hierzu zahlreiche Beispiele.

Ende des 19. Jahrhunderts galt Litauisch als „Bauernsprache“, so dass Kleinadel und gebildete Schichten Polnisch verwendeten. Auch in der Literatur dominierte Polnisch. Der Dichter Antanas Baranauskas (1835-1902) wechselte beispielsweise vom Polnischen zum Litauischen unter dem Einfluss von Karolina Praniauskaitè (1828-1859), die selbst ihre - heute größtenteils vergessenen - Gedichte fast ausschließlich auf Polnisch schrieb (Stoberski 1974: 55 f.). Wäre nicht der frühe Tod der Dichterin gewesen, hätte sie vermutlich auch zum Litauischen gewechselt. Neben Karolina Praniauskaitè beeinflusste auch Juliusz Słowacki, einer der Nationaldichter Polens, das Interesse Antanas Baranauskas für die Folklore Litauens ${ }^{8}$. Es war die Epoche der Romantik, die sich Märchen, Balladen, Volksliedern und der Volkssprache zuwandte. Baranauskas beschäftigte sich linguistisch mit litauischen Dialekten, übersetzte die Bibel ins Litauische und schrieb eigene Gedichte in litauischer Sprache.

8 Vgl. Antoni Baranowskis Biographie in polnischer Sprache: www.dziecionline.pl/ Suwalki/ludzie/baranowski.htm 
BLUM-BARTH

Als Meilenstein in der litauischen Literatur wird sein Gedicht „Anykščių šilelis“ bezeichnet. Es wurde 1861, knapp zwei Jahre nach seiner Entstehung, erstveröffentlicht und besingt die Schönheit der litauischen Wälder und die Verbundenheit der Menschen mit der Natur.

1896, sechs Jahre vor dem Tod Antanas Baranauskas, wurde bei Wilna, das heutige Vilnius, der weißrussisch-litauische Dichter Moische Kulbak, geboren, der spätestens 2017, als Litauen das Gastland der Leipziger Buchmesse war, wiederentdeckt wurde (vgl. Kulbak 2017). Kulbak zählt zu den größten Dichtern jiddischer Sprache seiner Zeit. Anfang der 20er Jahre des vergangenen Jahrhunderts war Wilna ein Zentrum der jiddischen Kultur. Dass Kulbak, der ursprünglich auf Hebräisch schrieb und neben Russisch, Litauisch und Polnisch auch Deutsch sprach, sich für Jiddisch als Literatursprache entschied, hatte ideologische Gründe.

Das bis 1904 anhaltende Verbot der zaristischen Behörden, Bücher in litauischer Sprache zu drucken, führte zur Popularisierung des Litauischen als Literatursprache und Entstehung der nationalen Romantik. Zu den bekanntesten Autoren litauischer Literatur der Zwischenkriegszeit zählen Balys Sruoga (1896-1947), Salomejja Nèris (1905-1945), Bernardas Brazdžionis (1907-2002). Wie der letztere gingen auch andere Schriftsteller - Henrikas Radauskas, Jonas Aistis, Alfonsas Nyka-Niliūnas - nach der deutschen und sowjetischen Besatzung Litauens ins Exil. Nach kürzeren Aufenthalten in Deutschland, Frankreich und der Schweiz zogen sie in die USA. Dort blieben sie der litauischen Sprache treu. Da sie fast alle Lyrik schrieben, war der Sprachwechsel ja sehr unwahrscheinlich. Im Exil beteiligten sie sich als Literaturkritiker und literarische Übersetzer. In der Heimat wurden sie diffamiert und tabuisiert, ihr Werk blieb bis zur Wiedererlangung der Unabhängigkeit Litauens weitgehend unbekannt.

Einer der bemerkenswertesten Autoren Litauens ist Jurgis Baltrušaitis (1873- 1944), der ebenfalls ins Exil ging, nach Frankreich, und dort vom Russischen zum Litauischen wechselte. Baltrušaitis wurde in einem kleinen Dorf im Nordwesten Litauens, der damals zum Russischen Reich gehörte, geboren. Während seines Physik- und Mathematikstudiums an der Universität Moskau lernte er die zentralen Figuren des russischen Symbolismus kennen: Konstantin Balmont, Waleri Brjussow und Wjatcheslaw Iwanow. Er debütierte als Lyriker 1899 und schrieb knapp 300 Gedichte in russischer Sprache. Sein erstes auf Litauisch verfasstes Gedicht erschien 1927. Sein Sprachwechsel war weniger politisch motiviert, er trug vielmehr einen experimentellen Charakter. Für einen Dichter ist es attraktiv und herausfordernd, einen neuen Trend oder Kunstgriff losgelöst von seinem Ursprung auszuprobieren. Der Hang des russischen Symbolismus zum Mystizismus führte zur Herausbildung von 
Symbolen, deren häufige Verwendung mit der Zeit zum Verlust ihres Gehalts und ihrer Wirkung führte. Anders war es in der litauischen Sprache, die diese Kunstrichtung noch nicht kannte und unverbrauchtes Sprachmaterial bot, das ein Dichter frei von Vorbildern und abseits der Muster gestalten konnte. Natürlich kannte Baltrušaitis diese Vorbilder und Muster im Russischen, aber sie dienten ihm im Litauischen als Empfehlung und nicht als Regel, die es zu befolgen galt. Dadurch entstand Freiraum zum Modifizieren, Ausprobieren, eben zum Experimentieren. Gerade dieses Moment ist ein Vehikel für den Sprachwechsel bei vielen Autoren, denn dadurch werden Kreativität und Inspiration generiert. Gleichzeitig findet Transfer von neuen literarischen Formen, Trends, Kunstrichtungen und Stilen aus einer Literatur in die andere statt. Wie fruchtbar der Sprachwechsel für Baltrušaitis war, zeigen seine drei Gedichtbände, ${ }^{9}$ die in Frankreich, fern vom litauischen Sprachraum, entstanden. Auch wenn sein erster Gedichtband in litauischer Sprache erst 1942, kurz vor seinem Tod, erschien, ging Baltrušaitis nicht nur als Symbolist, sondern als Erneuerer der litauischen Literatur in ihre Geschichte ein.

Als Sprachwechsler und zweisprachiger Autor ist Baltrušaitis ein Paradebeispiel für textexterne Mehrsprachigkeit. Seine literarischen Übersetzungen aus zahlreichen Sprachen ins Russische und Litauische offenbaren, wie breit und vielfältig das Spektrum seiner textexternen Mehrsprachigkeit ist. In seiner russischen Phase übersetzte er zahlreiche Dichter und Dramatiker ins Russische: George Gordon Byron, Henrik Ibsen, Knut Hamsun, Gerhart Hauptmann, Gabriele D’Annunzio, Oscar Wilde, Johan August Strindberg, Søren Kierkegaard sowie Rabindranath Tagore u. a. Baltrušaitis’ Leistung als literarischer Entdecker und Vermittler wird mit einem Preis honoriert, der seinen Namen trägt und seit 2006 verliehen wird.

Der erste Preisträger dieser Auszeichnung ist Georgi Efremow, ein russischer Dichter, Publizist und Übersetzer. Den Baltrušaitis-Preis erhielt Efremow für seine Übersetzungen der litauischen Autoren ins Russische. Seit 1975, als seine erste Übersetzung von Eduardas Mieželaitis erschien, übertrug Efremow praktisch die gesamte zeitgenössische litauische Lyrik ins Russische: Algimantas Baltakis, Bernardas Brazdžionis, Albinas Bernotas, Vladas Braziūnas, Sigitas Zigmas Geda, Marcelijus Teodoras Martinaitis, Justinas Marcinkevičius, Aidas Marčènas, Jonas Strielkūnas, Aivaras Veiknys u.a. ${ }^{10}$ Fast jeder der genannten Lyrikerinnen und Lyriker übersetzt auch selbst Lyrik

9 Ašarų Vainikas I, 1942; Ašarų Vainikas II, 1948; Aukuro dūmai, 1948. Gedichte aus diesen drei Bänden sind online zugänglich: www.tekstai.lt/tekstai/772-baltrusaitisjurgis.

10 Vgl. Online-Veröffentlichungen: https://magazines.gorky.media/authors/e/georgijefremov und www.vekperevoda.com/1950/efremov.htm. 
aus anderen Sprachen ins Litauische. Damit wird deutlich, wie verbreitet textexterne Mehrsprachigkeit gerade unter den Lyrikern ist. Es ist ein Phänomen, das über Litauen und das Baltikum hinausgeht und als typisch für Lyriker gelten kann. Das Akustische einer anderen Sprache - Melodie, Rhythmus, Klang - reizt und inspiriert eigene Spracharbeit, schult das Ohr, sensibilisiert für die Musikalität und bietet sich als Übungsraum an.

Auch wenn Georgi Efremow mit dem Baltrušaitis-Preis vordergründig für seine Popularisierung der litauischen Literatur ausgezeichnet wurde, darf nicht vergessen werden, dass er selbst ein Dichter ist, einer der bekanntesten Dichter Litauens, die auf Russisch schreiben. Den litauischen Lesern ist er jedoch bestens bekannt, da seine Lyrik von den von ihm übersetzten litauischen Dichtern aus dem Russischen ins Litauische übertragen wird.

Die Tradition der literarischen Übersetzung, insbesondere der Lyrikübersetzung, erhielt in Russland von den Dichtern des Silbernen Zeitalters ${ }^{11}$ vielfältige Impulse und wurde in der sowjetischen Zeit fortgesetzt. In russischer Sprache wurden Anthologien der Literatur fast aller sowjetischen Republiken herausgegeben. Gewiss waren diese Ausgaben politisch und ideologisch motiviert. Wie sonst erklärt man die zahlreichen Ausgaben der litauischen Literatur während des Zweiten Weltkrieges. ${ }^{12}$ Mit ihren Werken sollten die Autorinnen und Autoren die kommunistischen Ideen und sozialistischen Werte popularisieren. Auch nach dem Zweiten Weltkrieg erschien eine Reihe von Anthologien litauischer Literatur in russischen Übersetzungen: „Поэты

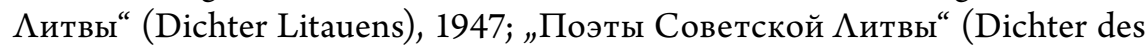
sowjetischen Litauens), 1948; „Аитовские новемиы“ (Litauische Novellen), 1948; „Поэзия Аитвы. Антология“ (Dichtung Litauens. Anthologie), 1950; „Проза Советской $\Lambda$ ивы“ (Prosa des sowjetischen Litauens), 1950, u. a.

Einer der meistübersetzten und bekanntesten Autoren des sowjetischen Litauens war Eduardas Mieželaitis. ${ }^{13}$ Der 1919 in Kareiviškis geborene Dichter war nicht nur in seiner Jugend ein aktiver Anhänger der kommunistischen

11 Dmitri Mereschkowskis Abhandlung „Über die Gründe des Verfalls der zeitgenössischen russischen Literatur“ (1893) inspirierte zahlreiche Dichter des Silbernen Zeitalters (Brjussow, Iwanow, Bunin, Achmatowa u. a.) zu Übersetzungen der europäischen und außereuropäischen Literatur ins Russische.

12 Auf Russisch erschienen: Anthologie der litauischen Prosa „Живая $\Lambda$ „ва“ (Lebendiges Litauen), 1942; „Вечная ненависть“ (Ewiger Наß), 1943; „За Советскую Прибалтику“ (Für das sowjetische Baltikum), 1943; „Аорога в Аитву“ (Der Weg nach Litauen), 1944, sowie Gedichtbände von Liudas Gira, Salomèja Nèris, Antanas Venclova und Kostas Korsakas.

13 Zur Biographie von Eduardas Mieželaitis sowie seinen Gedichten, vgl. www.tekstai.lt/ tekstai/288-miezelaitis-eduardas. 
Vom historischen Erbe zur selbstbestimmten Sprach(en)politik?

Ideen, sondern blieb Parteimitglied auch nach der Wende. Dabei wurde er 1946 „für die Ideenlosigkeit“ seiner Dichtung von Literaturfunktionären scharf kritisiert. Vermutlich um weniger Oden an die kommunistische Partei schreiben zu müssen, begann Mieželaitis für Kinder und Jugendliche zu dichten und übersetzte Werke anderer Autoren ins Litauische. Neben Puschkin und Lermontov übersetzte er auch Leonid Martynov aus dem Russischen, der seinerseits Mieželaitis Werke ins Russische übertrug. Für seine literarische Tätigkeit wurde er 1962 mit dem Leninorden der Sowjetunion ausgezeichnet. Im unabhängigen Litauen wurde er für seine politische Haltung zwar angefeindet, aber gleichzeitig gilt er bis heute als einer der größten litauischen Dichter des 20. Jahrhunderts.

Das gegenseitige Übersetzen ist unter den Lyrikern durchaus verbreitet. Der 1937 in Memel geborene Tomas Venclova, der seit 1977 als Dissident im US-amerikanischen Exil lebt, übersetzte den polnischen Dichter Czesław Miłosz und den russischen Joseph Brodsky, während die beiden seine Lyrik aus dem Litauischen übertrugen (vgl. Veser 2017: 14). ${ }^{14}$ In den USA schrieb Venclova nicht nur auf Litauisch, sondern auch auf Russisch. Diese Zweisprachigkeit war gewiss nicht politisch motiviert, sondern ergab sich aus seiner literarischen und literaturwissenschaftlichen Tätigkeit.

Die Literatur des heutigen Litauens orientiert sich am globalen Buchmarkt, der englischsprachig ist. Die Online-Zeitschrift Vilnius Review bietet eine beeindruckende Zahl an literarischen Texten der litauischen Gegenwartsliteratur und setzt sich deren Popularisierung zum Ziel. ${ }^{15}$ Dieses soll erreicht werden, indem literarische Texte in englischer Übersetzung dem breiteren Leserkreis zugänglich gemacht werden. Um das anfallende Arbeitspensum zu bewerkstelligen, wird ein Übersetzerteam beschäftigt. Ohne finanzielle Unterstützung sind solche Projekte kaum realisierbar. Als Sponsoren von Vilnius Review werden folgende Stiftungen genannt: Lithuanian Council for Culture, Association LATGA, City of Vilnius, modernaus meno centras, Spaudos, radijo ir televizijos remimo fondas. ${ }^{16}$ Es handelt sich also um staatliche Strukturen, die im Einklang mit der (Kultur)Politik der litauischen Regierung insbesondere seit 2010 vermehrt das Englische als Kommunikationssprache verwenden. Zum einen wird dadurch die Weltoffenheit Litauens deutlich, das sich im Zuge der Globalisierungsprozesse immer stärker in die europäische und Weltgemeinschaft integriert. Zum anderen geht damit die Bedeutung und

14 Siehe auch das Video, in dem Tomas Venclova ein Gedicht von Miłosz in polnischer Sprache und in eigener Übersetzung ins Litauische vorträgt: www.tekstai.lt/tekstai/374venclova-tomas/6233-tomas-venclova-skaito-czesawa-miosza-video-2011.

15 Vgl. http://vilniusreview.com/about.

16 Ebd. 
BLUM-BARTH

die Kenntnis des Russischen und des Polnischen verloren. Diese Sprachen sind ein wichtiger Teil der Geschichte Litauens, scheinen jedoch mehr der Vergangenheit anzugehören, als in der Zukunft eine wichtige Rolle zu spielen.

Dieser Pessimismus müsste allerdings angesichts der 2019 erschienenen dreibändigen Anthologie der russischen Dichtung Litauens (Антология русской поэзии Аитвы) relativiert werden. Sie bringt Texte von knapp 350 Autorinnen und Autoren, die seit Jahrhunderten mit Litauen verbunden waren und auf Russisch schrieben. ${ }^{17}$ Die Initiatoren dieser Anthologie sind Mitglieder von „Logos“, des Vereins der russischsprachigen Dichter Litauens. Inspiriert wurden sie von der Anthologie Russische Poesie Lettlands, die im Nachbarland erschien. Gleichzeitig setzt „Logos“ mit seiner dreibändigen Ausgabe ein Zeichen des Protests gegen die Sprach(en)politik im heutigen Litauen.

Seit seiner Unabhängig ist Litauen bemüht, Litauisch vor Einflüssen des Russischen sowie vor Anglizismen und nicht litauischen Wörtern zu schützen. Mit dieser Aufgabe sind zwei staatliche Behörden beauftragt, die Staatliche Kommission der litauischen Sprache und die Staatliche Sprach-Inspektion, die nicht nur mit Argusaugen, sondern mit Tadel, Verwarnungen und Geldstrafen bis zu 434 Euro für die Reinheit der litauischen Sprache sorgen sollen (Stašaitytė 2017). Als Richtlinie dient der „Sprachpolizei“, wie diese Behörden im Volksmund genannt werden, ein 25-seitiger Katalog der „großen Fehler der litauischen Sprache“, die insbesondere Autoren, Journalisten und öffentliche Funktionsträger nicht begehen dürfen. Die Leiterin der Sprachkommission, Daiva Vaišnienè, beteuert allerdings, dass für die Literatur „eine totale Freiheit“ gelte (Stašaitytė 2017). Der Roman Pietinia kronikas (2016) von Rimantas Kmitas, der in der Umgangssprache geschrieben wurde und mit Schimpfwörtern gespickt ist, soll als Beweis für die Freiheit der Literatur im heutigen Litauen herhalten (Stašaitytė 2017). Allerdings ist bemerkenswert, dass eine solche Sprachzensur wie in Litauen in den anderen postsowjetischen Staaten nicht praktiziert wird - mit Ausnahme von Russland. Die Sprachpolitik Litauens erklärt Heiko F. Marten zutreffend mit der postkolonialen Ausgangssituation: „Die durch die postkoloniale Ausgangssituation begründete Sprachpolitik führt zu einigen Praktiken, die im internationalen Vergleich ungewöhnlich sind.“ (Marten 2017: 217).

${ }_{17}$ Ausführlich zur Vorstellung dieser Anthologie, vgl. den Zeitungsartikel: В мире русской Аитературы Аитвы - революция. In: Обзор Nr. 1197. Abrufbar unter: www.obzor.lt/news/n55785.html (29.06.2020). 
Vom historischen Erbe zur selbstbestimmten Sprach(en)politik?

\section{Sprach(en)politik und literarische Mehrsprachigkeit in Lettland}

Eine „postkoloniale Ausgangsposition“ gilt auch für Lettland. Die Eroberung seiner Gebiete durch den Deutschen Orden und die Hanse, die schwedische Herrschaft im 17. Jahrhundert, die Besetzung durch das Russische Reich Anfang des 18. Jahrhunderts und die sowjetische Besatzung im Juni 1940 sind Höhepunkte der postkolonialen Vergangenheit Lettlands. Im Spätmittelalter werden von der Bildungsschicht Latein und Deutsch verwendet. Erst im 19. Jahrhundert, als der Einfluss des Deutschen nachlässt, entsteht Literatur in lettischer Sprache. Die Epoche der Romantik weckt das Interesse für Volkslieder und Balladen, die gesammelt und studiert werden (Krišjānis Baron). Die in lettischer Sprache verfassten Prosawerke jener Zeit entstehen unter dem Einfluss der westeuropäischen und insbesondere der deutschen Literatur. Die lutheranischen Pastoren übersetzen zahlreiche Werke aus dem Deutschen, um so eine Alternative zu freiheitsliebenden lettischen Volksliedern und Volksmärchen zu schaffen. Unter den lettisch-deutschen Schriftstellern ist u. a. Andrievs Niedra (1871-1942) zu nennen. Ferner sei auch auf Rūdolfs Blaumanis (18631908), einen der bekanntesten lettischen Schriftsteller, hingewiesen. Nach dem Abschluss der deutschen Handelsschule in Riga schrieb Blaumanis seine ersten Publikationen auf Deutsch. Seine Novellen und Kurzgeschichten verfasste er auf Lettisch. Viele von ihnen, wie z. B. das Drama „Die Indrans“ (1904) übersetzte er dann ins Deutsche (Kalnačs/Füllmann 2017). Später wurde er Mitglied der sozialdemokratischen Bewegung die „Neue Strömung“. Ende des 19., Anfang des 20. Jahrhunderts lässt sich eine immer größere lettische Bildungsschicht für Ideen des Sozialismus begeistern. Unter ihnen war auch das bekannteste literarische Paar Lettlands Aspazija (1865-1943) und Rainis (1865-1929). Ihre ersten Gedichte schrieb Aspazija auf Deutsch, wechselte aber bald zum Lettischen. Zusammen mit Rainis, dem wichtigsten Dichter Lettlands, übersetzte sie Goethes „Faust“ ins Lettische. Für seine sozialistische Weltanschauung wurde Rainis 1897 nach Pskow und Slobodskoi verbannt. In den fünf Jahren seiner Zwangsverschickung schrieb er nicht nur Gedichte in lettischer Sprache, sondern übersetzte Schiller, Heine, Shakespeare und Puschkin ins Lettische. Der Einfluss der deutschen Sprache und Literatur auf die lettische Literatur ist noch Anfang des 20. Jahrhunderts nicht abgeebbt.

Der Geschichte der literarischen Mehrsprachigkeit in Lettland widmet sich Alexander Zapol in der Einleitung zu der von ihm 2011 herausgegebenen Anthologie (Zapol 2011), die einen langen Abschnitt der lettischen Lyrik von 1680 bis 2010 anhand der Texte von 40 Autorinnen und Autoren vorstellt. Zapol hebt u. a. hervor, dass Ende des 19. und Anfang des 20. Jahrhunderts viele lettische Autorinnen und Autoren sich an Vorbildern der russischen, deutschen, französischen und skandinavischen Literaturen orientierten, aus diesen 
Sprachen übersetzten und auch selbst ihre Texte auf Deutsch oder Russisch schrieben. Dies hing sowohl mit den historischen Rahmenbedingungen zusammen (Schulunterricht fand in deutscher, später in russischer Sprache statt), als auch mit der Orientierung an neuen literarischen Tendenzen und Prozessen, die in diesen Literaturen entstanden. Es war kein Widerspruch, ein lettischer Autor zu sein und in russischer oder deutscher Sprache zu schreiben. Die in russischer Sprache verfassten Gedichte von Aleksandr Čak entstanden beispielsweise nach seinem Treffen mit Wladimir Majakowski und ahmen den Stil des russischen Futurismus nach. Gleichzeitig knüpfen seine auf Lettisch geschriebenen Gedichte an die Tradition der europäischen Großstadtlyrik an.

Mit dem Ausbruch des Zweiten Weltkriegs wurde die Bevölkerungsstruktur Lettlands grundlegend verändert: Anfang Oktober 1939 wurde Lettland gezwungen, die Stationierung von 25000 sowjetischen Soldaten auf lettischem Gebiet zuzulassen. Kurz darauf ließ Hitler 50000 Deutschbalten umsiedeln (vgl. Garleff 2002). Am 17. Juni 1940 rollten sowjetische Panzer durch die Straßen Rigas, und ca. 100000 Rotarmisten befanden sich auf lettischem Territorium. Zwischen 1940 und 1990 stieg der Anteil der Russen in der ethnischen Zusammensetzung der lettischen Bevölkerung auf 34 \%. Während der sowjetischen Herrschaft „sank der Anteil ethnischer Lett/-innen an der lettischen Bevölkerung bis 1989 auf 52 Prozent.“ (Mierina 2020) Das in der Sowjetzeit russifizierte Lettland zeigt sich nach der Wende weniger bemüht um die Bekämpfung der russischen Sprache als dies z. B. in Litauen der Fall ist.

Anders als in Litauen schreiben in Lettland sehr viele Autoren auf Russisch, allerdings sind dies hauptsächlich ethnische Russen. Ausschlaggebend für ihre Tätigkeit ist die 1999 gegründete Textgruppe „Orbita“. Ihre Arbeiten zeichnen sich durch das Experimentieren mit anderen Medien aus (Photographie, Musik, Film, Graphik, Malerei). Von den zahlreichen Aktivitäten der Vereinigung wird die Herausgabe zweisprachiger Lyrikbände besonders betont: „Orbita has [...] produced a number of bilingual (Russian-Latvian) poetry collections and publications; issued an anthology of contemporary Russian poetry in Latvia - a unique study of this phenomenon" ${ }^{18}$ Die Mitglieder der Gruppe ${ }^{19}$ (Sergej Timofejew, ${ }^{20}$ Artur Punte, Alexander Zapol, Semjon Chanin, Zhorzh Uallik u.a.) zeigen sich weltoffen und nehmen an Literatur-Events im In- und Ausland teil. Auch wenn Russisch die Sprache ihres kreativen Schaffens ist, spielen Lettisch und Englisch eine wichtige Rolle in ihrem Schaffensprozess.

18 www.orbita.lv

19 Vgl. zur Liste der Autoren http://archive.orbita.lv/authors. Beim Anklicken auf den Namen erscheinen ausgewählte oder aktuelle Texte.

20 Weitere Gedichte findet man in den Online-Magazinen Vavilon: www.vavilon.ru/ texts/index.html und textonly http://textonly.ru/authors/?issue $=15$ 
Die zweisprachigen Gedichtausgaben, die „Orbita“ initiiert, stellen das russische Original und die lettische Übersetzung nicht einfach nebeneinander, sondern inszenieren beide Sprachen, indem sie ihren Klang häufig ins Zentrum rücken. Beispiele dafür sind nicht nur zweisprachige Lesungen, Performances und multimediale Projekte, die häufig mit Sound experimentieren, sondern auch die Gestaltung von Buchcover, wie etwa des Gedichtbandes Stereo (2012) von Sergej Timofejew und die Fokussierung auf den Klang der beiden Sprachen, wie bereits im Titel der Gedichtbände Tрансферы/ Transfēri (2013) und Алчность/Alkatība (2019) von Jelena Glazova. ${ }^{21}$ Auch wenn es überzogen wäre anzunehmen, dass die auf Russisch schreibenden Autoren Lettlands den performativen Soundformaten dank ihrer textexternen Zweisprachigkeit zu einem so hohen Stellenwert verholfen haben, ist es nicht von der Hand zu weisen, dass das Moment der akustischen Gegenüberstellung bzw. des Zusammenspiels zwischen dem russischen Original und der lettischen Übersetzung beabsichtigt ist.

Der Band Вижу слышу молчу/Redsu dzirdu kluseju (2013) von einem 1975 geborenen Autor, der unter dem Pseudonym Zhorzh Uallik schreibt, präsentiert Texte, die nach akustischem bzw. phonetischem Prinzip konzipiert sind. Ihnen liegen Alliterationen, akustische Assoziationen, phonetische Verwandtschaft, Echo-Effekte sowie Spiegelungen und Doppelungen zugrunde. ${ }^{22}$ Stellenweise entfalten die Gedichte dieses Bandes eine psychodelische Wirkung, die durch die Überreizung der auditiven Wahrnehmung erzielt wird. Der Band ist als Doppelband erschienen und bringt die Übertragung dieser Gedichte ins Lettische. Die beiden Bände lesen sich je von einer Seite, wobei sie mit dem gemeinsamen Rückcover verbunden sind. Diese aufwendige innovative Gestaltung des Buches deutet auf die herausragende Bedeutung der Sprachen und ihr Zusammenspiel sowohl bei der Entstehung der Gedichte als auch bei ihrer Übertragung ins Lettische hin.

${ }^{21}$ Auf der Webseite der Autorin sind neben Texten auch ihre Performances einzusehen: www.jelena-glazova.com/texts/

22 Kirill Korchagin verortet Ualliks Texte in der Tradition der russischen Konkretisten: „Behind each poem there is a kind of an ecstatic, capturing gesture in which he uses awkward word combinations that serve to bring the entire mass of the poem to an 'aroused' state. Uallik flirts with naive poetry and primitivism, balancing on the edge of art brut, coming into contact with the necroinfantile experiments of Yuri Odarchenko and the oberiu group of Russian avantgardists. All this brings the author close to the Russian post-avant-garde (the school of Sergei Buryukov) and distinguishes him from other members of Orbita." https://postnonfiction.org/wp-content/uploads/20 18/05/ orbita-catalog-screen-1.pdf, S. 13. 
BLUM-BARTH

Die enge Zusammenarbeit zwischen den auf Russisch schreibenden Autorinnen und Autoren und Übersetzerinnen und Übersetzern ins Lettische, die ihrerseits selbst Dichter sind, die auf Lettisch schreiben, generiert nicht nur Synergien und Kreativität, sondern auch Experimentierfreude. Artur Punte, einer der Redakteure der zweisprachigen Ausgaben, brachte 2014 seinen Gedichtband Поэтические посвящения/Poētiskie veltijumi heraus, in dem zwischen russischen Originalen und ihren Übersetzungen ins Lettische auch Gedichte stehen, die Punte auf Lettisch verfasste. Auch wenn dies noch lange nicht bedeutet, dass Punte in der zweiten Sprache seines kreativen Schaffens den gleichen literarischen Rang hat, zeigt sich jedoch, dass Versuche, in einer anderen Sprache Literatur zu schreiben, einen experimentellen Charakter haben, dem durchaus spielerische Momente und Kreativität generierende Absichten zugrunde liegen. Gleichzeitig wird deutlich, dass der literarische Text nicht frei vom Einfluss der textexternen Mehrsprachigkeit bleibt. Er manifestiert sich vordergründig in der auditiven Gestaltung der Sprache, in der akustischen Wahrnehmung des Textes sowie in sprachvergleichenden, sprachkonsolidierenden oder sprachkontrastierenden Elementen, sowie in den vielfältigen onomatopoetischen Stilmitteln, worauf auch Ilva Skulte verweist:

Reading Punte, it seems the poet speaks following a developmental logic of events, conversations or associations, but then suddenly changes course, remaining under the disguise of language: what comes to the foreground is a linguistic interplay, transfers, breaks, sound repetitions, rhythm and metre. It is as if the poetry emerges from the story and language (or vice versa) in front of our eyes, thanks to its special, textually tangible form, a form that can express itself in tonality. ${ }^{23}$

Die Zweisprachigkeit und das Zusammenspiel der Sprachen werden auch durch das Design des Bandes Вплавь/Peldus von Semjon Chanin betont. Magnete wurden eingesetzt, um so die Anziehung zwischen dem Band mit russischen Originalen und dem Band mit Übersetzungen ins Lettische eindrucksvoll zu veranschaulichen. Die Übersetzungen wurden von Dichtern wie Kārlis Vērdiņš, Pēteris Draguns, Liana Langa, Jānis Rokpelnis, Marts Pujāts, Jānis Elsbergs u. a. vorgenommen. Gedichte einiger von ihnen sowie von Eduards Aivars, Edvīns Raups, Māris Salējs übersetzt Semjon Chanin aus

23 Ilva Skulte zum Band Поэтические посвящения/Poētiskie veltijumi im Verlagskatalog: https://postnonfiction.org/wp-content/uploads/2018/05/orbita-catalog-screen-1.pdf 
dem Lettischen ins Russische. Ferner überträgt er auch aus dem Englischen, etwa den amerikanischen Dichter russischer Herkunft Eugene Ostashevsky. ${ }^{24}$

Die Gegenwartsliteratur Lettlands - unabhängig ob in lettischer oder in russischer Sprache - entsteht vor dem Hintergrund der gesellschaftlichen Zweisprachigkeit. Dies führt zur Herausbildung verschiedener Formen der literarischen Mehrsprachigkeit. Diese existieren sowohl in textexternen als auch textinternen Bereichen. Neben literarischen Übersetzungen, die für die textexterne Mehrsprachigkeit lettischer Gegenwartsautorinnen und -autoren kennzeichnend ist, scheint zunehmend die textinterne Mehrsprachigkeit eine wichtige Rolle zu spielen. Dabei ist zu beobachten, dass nicht so sehr manifeste als vielmehr latente Mehrsprachigkeit diese Texte kennzeichnet. Interferenz der Sprachen (z. B. russische Syntax in der lettischen Sprache), Lehnübersetzungen, Sprachlatenz und interlinguales Wortspiel scheinen die häufigsten Formen der latenten Mehrsprachigkeit (Blum-Barth 2021) in der lettischen Gegenwartsliteratur zu sein. In diese Richtung geht auch die Bewertung der beabsichtigten Sichtbarkeit und der Wechselwirkung des Russischen und Lettischen in zweisprachigen Ausgaben der „Orbita“ durch den Dichter und Kritiker Kirill Kortchagin als „utopisches Projekt der gemeinsamen lettisch-russischen poetischen Sprache" (Kortchagin 2015). ${ }^{25}$

\section{4 Übersetzungen im Kontext literarischer Mehrsprachigkeit}

Sieht man von traditionellen Volksliedern und -märchen ab, so entstand die Literatur Litauens wie auch Lettlands Anfang des 19. Jahrhunderts vor einem mehrsprachigen Hintergrund. Historisch bedingt übte der Einfluss der polnischen Sprache und Literatur in Litauen sowie der deutschen in Lettland einen großen Einfluss auf die Autorinnen und Autoren des jeweiligen Landes aus. Viele unternahmen ihre ersten literarischen Schreibversuche nicht in der Muttersprache, sondern auf Polnisch bzw. auf Deutsch, die Schulunterrichtssprache im jeweiligen Land waren. Gleichzeitig spielten Orientierung an westeuropäischer, skandinavischer und russischer Literatur, die sich nicht zuletzt in der Übersetzung manifestierte, eine wichtige Rolle bei der Entstehung der nationalen Literatur Litauens und Lettlands.

${ }^{24}$ Als Übersetzter verwendet Chanin seinen bürgerischen Namen Alexandr Zapol. Hier die Kurzbiographie des Autors in englischer Sprache: http://latvianliterature.lv/en/ translators $/ 80$.

25 Im Original lautet das Zitat: „[...] читатель сталкивается со своего рода утопическим проектом общего матышско-русского поэтического языка“. 
Die sowjetische Epoche stellte beide Länder vor vergleichbare Herausforderungen - Russifizierung und Ideologisierung -, die in Litauen und Lettland im Vergleich zu anderen sowjetischen Republiken noch moderat ausfielen. Im Unterschied zu Autorinnen und Autoren anderer sowjetischer Republiken waren bei litauischen und lettischen Autorinnen und Autoren weder Sprachwechsel noch Zweisprachigkeit, die häufig politisch-ideologisch motiviert sind, geläufige Phänomene. In der literarischen Mehrsprachigkeit dominiert ihre textübergreifende/textexterne Form.

Für die Erforschung der literarischen Mehrsprachigkeit, insbesondere der Übersetzung als eines der Formate der textexternen Mehrsprachigkeit lassen sich sowohl in Litauen als auch in Lettland während der sowjetischen Epoche zwei interessante Aspekte beobachten: 1. Die Gründe für Übersetzungen und 2. die konsultierende Funktion der russischen Sprache.

1. Literarische Übersetzung war eine Option, literarisch tätig zu sein, wenn Autorinnen und Autoren ein Schreib- und Veröffentlichungsverbot erhielten, ideologische Instrumentalisierung ihrer Werke verweigerten oder mit ihrer literarischen Tätigkeit den Lebensunterhalt bestreiten wollten.

2. Bemerkenswert ist dabei, dass auffallend viele Autorinnen und Autoren aus sehr vielen verschiedenen Sprachen übersetzten. Ohne ihre Fremdsprachenkenntnisse schmälern oder hinterfragen zu wollen, muss vermutet werden, dass die russische Sprache im Übersetzungsprozess eine Vermittlungsrolle spielte. Anders gesagt: dem Russischen kam die sogenannte konsultierende Funktion zu. Auf Russisch lagen mehrere Übersetzungen der Klassiker der Weltliteratur vor und übertrafen sich gegenseitig in ihrer hohen künstlerischen Qualität, so dass ihre Hinzuziehung bei der Übersetzung in eine andere Sprache eine vergleichende Vorgehensweise ermöglichte und viele Übersetzungsentscheidungen erleichterte. Diesen Status behält Russisch immer noch im Prozess der literarischen Übersetzung in die Sprachen der ehemaligen Sowjetrepubliken und der Ostblockstaaten bei. Ähnlich konsultierend können auch Englisch, Spanisch, Französisch u. a. herangezogen werden, um eine Übersetzungsentscheidung zu treffen. Dadurch wird deutlich, dass Sprachen an textexterner literarischer Mehrsprachigkeit sowohl aktiv als auch passiv beteiligt sein können. Besonders brisant sind diese Aspekte bei der Übersetzung der lyrischen Texte. Da die Übersetzung von Lyrik durch einen Übersetzer, der selbst Lyriker ist, eine Art Nimbus verliehen bekommt und ein höheres Ansehen genießt, ${ }^{26}$ kommt es öfter vor, dass Dichter auch aus Sprachen übersetzen, die sie nur begrenzt sprechen. Man arbeitet dann mit Interlinearübersetzungen, eine dem Ausgangstext strukturgetreue

26 Z. B. Übersetzungen von Paul Celan, Hans Magnus Enzensberger u. a. 
Vom historischen Erbe zur selbstbestimmten Sprach(en)politik?

Wort-für-Wort-Übersetzung, die auch verschiedene Entsprechungen einzelner Wörter angibt.

Trotz des gleichen sowjetischen Erbes entschieden sich Litauen und Lettland nach der Erlangung ihrer Unabhängigkeit für eine sehr unterschiedliche Sprach(en)politik. Ohne noch weiter auf ihre Unterschiede einzugehen, sei hier ihre Gemeinsamkeit hervorgehoben: eine rege literarische Übersetzungstätigkeit. Dabei dominiert die Bemühung, litauische bzw. lettische Autorinnen und Autoren durch Übersetzungen zu popularisieren. Während litauische Gegenwartsautorinnen und -autoren ins Englische übersetzt werden (etwa in der Online-Zeitschrift Vilnius Review), widmet sich in Lettland die Textgruppe „Orbita“ der Herausgabe der zweisprachigen russisch-lettischen Werkausgaben ihrer Gruppenmitglieder. Da solche Projekte ohne finanzielle Förderung langfristig nicht überlebensfähig sind, lassen sich an den staatlichen Geldgebern sprachpolitische Ziele und Interessen ablesen.

\author{
Natalia Blum-Barth \\ natalia.blumbarth@uni-mainz.de \\ University of Mainz \\ GERMANY
}

\title{
Literaturverzeichnis
}

Blaumanis, R. 2017. Frost im Frühling. Die deutschsprachigen Erzählungen. Vollständige Ausgabe aller vom Autor selbst auf Deutsch verfassten Werke. Herausgegeben von Benedikts Kalnačs und Rolf Füllmann. Bielefeld: Aisthesis.

Blum-Barth, N. 2019. Literarische Mehrsprachigkeit. Versuch einer Typologie. Spiegelungen. Zeitschrift für deutsche Kultur und Geschichte Südosteuropas, 2.19, 9-24.

Blum-Barth, N. 2021. Poietik der Mehrsprachigkeit. Theorie und Techniken des multilingualen Schreibens. Heidelberg: Winter.

Borisova, I. Yu. Osobennosti sovetskoj politiki s $1917 \mathrm{~g}$. do konca 1930-h g. = Борисова, И. Ю. Особенности советской языковой политики с 1917 г. до кониза 1930-х 2. Veröffentlicht auf der Webseite der Staatlichen Universität Sankt Petersburg, http://rus-gos.spbu.ru/index.php/article (29.06.2020).

Chalitova, A. M. 2010. Yazykovaya politika v SSSR v 1920-1930 gg. Regional'nyj opyt. - Vestnik Baškirskogo universiteta, 15 (1), 155-157. = Хамитова, А. М. 2010. Языковая политика в СССР в 1920-1930 гг. Региональный опыт. - Вестник Башкирского университета, 15 (1), 155-157, https://cyberleninka.ru/article/n/ yazykovaya-politika-v-sssr-v-1920-1930-gg-regionalnyy-opyt (30.06.2020).

Garleff, M. 2002. Die Deutschbalten als nationale Minderheit in den unabhängigen Staaten Estland und Lettland. - G. von Pistohlkors, Hrsg., Deutsche Geschichte im Osten Europas. Baltische Länder. Berlin: Siedler Verlag, 452-550. 
Gellhaus, A., Hrsg. 1997. „Fremde Nähe“. Celan als Übersetzer. Eine Ausstellung des Deutschen Literaturarchivs in Verbindung mit dem Präsidialdepartement der Stadt Zürich im Schiller-Nationalmuseum Marbach am Neckar und im Stadthaus Zürich. Marbach am Neckar: Deutsche Schillergesellschaft.

Kortchagin, K. 2015. „Identičnosti net“. Poety postsovetskogo prostrantsva na perekrestke kul'tur i yazykov. - Novyj mir, 1. = Корчагин, К. 2015. „ИАентичности нет“. Поэты постсоветского пространства на перекрестке культур и языков. Новыц Мир, 1, www.litkarta.ru/dossier/korchagin-kirill-identichnosti-net/ dossier_6231/(30.06.2020).

Kostiucenko, A. 2016. Sprachen und ihre Sprecher in Litauen: Eine soziolinguistische Untersuchung zum sozialen Status des Litauischen, Polnischen und Russischen. Berlin: Logos.

Kremnitz, G. 2015. Mehrsprachigkeit in der Literatur. Ein kommunikationssoziologischer Überblick. 2., erw. Auflage. Wien: Praesens Verlag.

Kulbak, M. 2017. Childe Harold aus Disna. Gedichte über Berlin. Berlin: Edition.fotoTAPETA Berlin.

Marten, H. F. 2016. Sprach(en)politik. Eine Einführung. Tübingen: Narr.

Mierina, I. 2020. Lettland - Auswanderung, Einwanderung, Diaspora. Abrufbar unter: www.bpb.de/gesellschaft/migration/laenderprofile/308741/lettland (Stand 6/7/2020).

Rudnev, D. V. 2007. Yazykovaya politika v SSSR i Rossii: 1940-2000-e gg. Gosdarstvennaya yazykovaya politika: problemy informacionnogo $i$ lingvističestnogo obepečeniya. SPb.: Filologičeskij fakul'tet SPbGU, 120-138. = РуАнев, А. В. 2007. Языковал политика в СССР и России: 1940-2000-е гг. Государственнал языковая политика: проблемы информационного и мингвистического обепечения. СПб.: Фимологический факультет СПбГУ, 120-138, http://rus-gos.spbu.ru/ index.php/article (30.06.2020).

Schreiner, P. 2006. Staat und Sprache in Europa: Nationalstaatliche Einsprachigkeit und die Mehrsprachenpolitik der Europäischen Union. Frankfurt am Main: Peter Lang.

Stašaitytè, V. 2017. Sprachpfleger mit „Schießgewehr“. Abrufbar unter: www.mdr.de/ nachrichten/osteuropa/ostblogger/litauen-sprachpolizei-100.html (30.06.2020).

Stoberski, Z. 1974. Historia literatury litewskiej: zarys (Lingusitics Poland). Wrocław: Zaklad Narodowy imienia Ossolinskich.

Veser, R. 2017. Freiheitskampf. Der litauische Dichter Tomas Venclova wird achtzig. Frankfurter Allgemeine Zeitung, 11. September.

Zapol, A. 2011. Latviešu krievu dzeja. Latviešu dzejnieku krievu valodā rakstīti dzejoḷi/

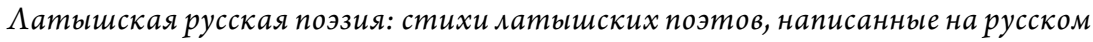
языке (Lettisch/Russsiche Lyrik. Auf Russisch geschriebene Gedichte lettischer Autoren). Riga: Neputns. 